\title{
Estudo preliminar do SISCOLO-Qualidade na rede de saúde pública de São Paulo
}

\section{SISCOLO-quality control system in the health public laboratories: preliminary study}

Marina Yoshiê Sakamoto Maeda'; Celso di Loreto'; Elci Barreto²; Maria José Cavaliere'; Maria Lucia Utagawa'; Yuriko Ito Sakai'; Romualdo Osório Corrêa ${ }^{2}$; Pekie Johanna Diaz Adura ${ }^{1}$; Valéria de Oliveira Marzola ${ }^{1}$

unitermos

Controle de qualidade externo

SISCOLO

Citologia cervicovaginal

\section{resumo}

Analisar criticamente os primeiros resultados do Sistema de Informação Laboratorial do Programa Nacional de Combate ao Câncer de Colo Uterino (SISCOLO-Qualidade). Foram utilizados esfregaços cervicovaginais corados pelo método de Papanicolaou, provenientes da rotina diagnóstica do Laboratório de Anatomia Patológica da Fundação Oncocentro de São Paulo (FOSP) e referentes a março de 2000. Esses casos foram reavaliados em estudo cego pela equipe do Setor de Citologia Oncótica da Divisão de Patologia do Instituto Adolfo Lutz/São Paulo. O Programa SISCOLO-Qualidade seleciona, entre os casos analisados no período, todas as citologias positivas (ASCUS, AGUS, NIC e carcinomas) e, para completar $10 \%$ do total das lâminas, todos os casos insatisfatórios e negativos aleatoriamente. Dos 8.370 casos da rotina da FOSP, o SISCOLO-Qualidade selecionou 837 amostras. Entre as amostras selecionadas, 23,4\% apresentaram material representativo da junção escamocolunar, 5\% foram considerados material insatisfatório e $71,1 \%$, material satisfatório, mas limitado. O resultado da análise foi $86,62 \%$ de concordância, $12,42 \%$ de discordância de um grau e $0,96 \%$ de discordância de mais de um grau. Concluímos que o Programa SISCOLO-Qualidade é aplicável na rede de saúde pública para monitoramento externo, atendendo às expectativas de qualidade exigidas pelo Ministério da Saúde.
The aim of the present work was to analyses critically the preliminary results of the computerized Quality System of gynecologycal cytology, implanted by the Public Health Ministry of Brazil - SISCOLO. Cervicovaginal smears stained by the Papanicolaou method were utilized. These smears were obtained through the diagnostic routine undertaken at the Oncocentro Foundation Pathology Laboratory of pathology, comprising cases of March 2000. Such cases were rescreened in a blind study, by the staff of the Pathology Division of the SP Adolpho Lutz Institute's Cytology Section. The SISCOLO-Quality Program selects, among all cases analyzed in the period, all such positive cases (ASCUS, AGUS, CIN and carcinomas) and to complete 10\% of all cases it selects all non-satisfying and negative cases at random. From the 8,370 cases selected in March 2000, the SISCOLO-Quality program selected 837 samples. Among the selected samples, 23,4\% showed squamo-columnar junction material, 5\% were considered non-satisfactory for diagnosis and $71.1 \%$, satisfactory although limited. The rescreening analysis showed concordance in $86.62 \%$ of the cases, one grade discordance was observed in $12.42 \%$ and two or more grades discordance in $0.96 \%$ of the cases. We concluded that the SISCOLO-Quality Program has applicability in the health public system as external monitoring method and meets the Health Ministry's expectations. key words

External quality control

SISCOLO

Cervicovaginal cytology 


\section{Introdução}

O programa de combate ao câncer de colo de útero, baseado em triagem por citologia cervicovaginal, representa uma estratégia eficaz para a redução da morbidade e da mortalidade provocadas por essa doença. Entretanto, nenhum teste de screening tem $100 \%$ de sensibilidade e especificidade; resultados falsos positivos e falsos negativos podem ocorrer ${ }^{(7,9)}$.

A promoção de garantia de qualidade continua sendo o maior objetivo da Academia Internacional de Citologia (IAC) e as estratégias para melhoria de qualidade diagnóstica em citologia têm sido motivo para vários estudos( ${ }^{(8)}$. No intuito de avaliar os profissionais envolvidos no diagnóstico citopatológico, várias estratégias de controle de qualidade interno têm sido propostas. A revisão aleatória de pelo menos $10 \%$ dos casos negativos ${ }^{(4,9,12)}$, o screening rápido ${ }^{(6,10)}$ e as análises de $100 \%$ das pacientes e dos casos a partir de parâmetros preestabelecidos têm sido utilizados rotineiramente no programa de controle de qualidade interno em citologia ginecológica do Instituto Adolfo Lutz ${ }^{(1)}$. Entretanto, a avaliação do desempenho dos diversos laboratórios somente pode ser alcançada pelo controle externo de qualidade ${ }^{(3)}$.

Em 1999, o Programa Nacional de Controle do Câncer do Colo do Útero (PNCCCU) estabelece normas e recomendações para rotina de monitoramento externo da qualidade. A partir de janeiro de 2000, os exames realizados por esses laboratórios passaram a ser informados através do Sistema de Informação Laboratorial do Programa Nacional de Combate ao Câncer de Colo Uterino (SISCOLO), formando um banco de dados em nível estadual. Através deste e da rotina específica do sistema, é realizada a escolha das lâminas a serem revistas. O tamanho da amostra, definido pelo sistema, é estabelecido, devendo atingir no mínimo $10 \%$ do total de exames realizados.

O objetivo do presente estudo é avaliar a aplicabilidade de um controle de qualidade que seja efetivo para monitoramento dos laboratórios da rede de saúde pública do estado de São Paulo e que tenha potencial para ser utilizado também em outros estados.

\section{Material e método}

O estudo de 837 casos foi proveniente de um banco de dados da Fundação Oncocentro de São Paulo (FOSP). O tamanho da amostra, definido pelo sistema SISCOLOQualidade, obedeceu como critério de seleção todas as lâminas de casos positivos e insatisfatórias e mínimo de 5\% dos exames normais, selecionados aleatoriamente, devendo atingir no mínimo $10 \%$ do total de exames realizados. Pelo critério de seleção foram selecionados 614 negativos, 93 ASCUS/AGUS, 114 NIC 1, oito NIC 2, quatro NIC 3, um carcinoma invasor e três insatisfatórios.

Para avaliação de ocorrência de falsos positivos ou falsos negativos foi utilizada a classificação de erros diagnósticos em citopatologia, proposta pela equipe do Ministério da Saúde do Canadá(2, 13):

a) falso negativo: diagnóstico inicial de material inadequado, citologia normal ou inflamatória e revisão com diagnóstico de NIC 3 ou carcinoma invasor;

b) falso positivo: diagnóstico inicial de $\mathrm{NIC}$ e/ou carcinoma invasor quando a revisão levou à conclusão diagnóstica de citologia normal ou inflamatória.

Com relação à amostragem foram consideradas:

- satisfatórias: quando havia presença de células da junção escamocolunar (JEC) e os esfregaços não eram limitados por fatores como esfregaço hemorrágico, purulento, espesso ou parcialmente dessecado;

- satisfatórias mas limitadas: quando não havia representação da JEC ou o esfregaço era limitado por algum dos fatores citados acima;

- insatisfatórias: os esfregaços com material escasso, hemorrágicos em excesso, purulentos ou dessecados, ou ainda extremamente espessos.

Os critérios para diagnóstico utilizados na revisão foram os descritos a seguir ${ }^{(11)}$.

\section{Amostra insatisfatória}

Composta por menos de $10 \%$ de células epiteliais ou amostra com células epiteliais obscurecidas $75 \%$ ou mais na superfície do esfregaço por material hemorrágico, purulento, dessecado ou constituído por áreas espessas.

\section{Citologia normal}

Amostra sem células anormais, com predomínio de células epiteliais escamosas; presença de células colunares em pequeno número, muco cervical e flora bacteriana, leucócitos e hemácias em pequena quantidade.

\section{Citologia inflamatória}

Presença de mutações celulares benignas (reativas e/ou reparativas). Essas alterações podem ocorrer tanto no citoplasma como no núcleo. No primeiro observa-se 
vacuolização, policromasia, pseudo-eosinofilia e halo perinuclear. No segundo podem ser vistas discretas hiper ou hipocromasia, cariomegalia, anisonucleose, picnose, cariorrexe e cariólise. A cromatina é fina e homogênea, granulosa ou com numerosos cromocentros e o contorno nuclear é liso, arredondado e uniforme.

\section{ASCUS e AGUS (atipia de significado indeterminado em células epiteliais escamosas ou glandulares)}

Presença de anormalidades mais acentuadas que aquelas atribuídas às alterações reativas, mas que qualitativa ou quantitativamente são insuficientes para definir um diagnóstico de lesão intra-epitelial ou carcinoma. Apresenta as seguintes características nucleares: núcleo aumentado, variação no tamanho e na forma, binucleação, hipercromasia nuclear, cromatina fina e distribuída regularmente.

\section{NIC 1}

Presença de células epiteliais escamosas superficiais ou intermediárias com aumento do volume nuclear, carioteca discretamente espessada, hipercromasia, nucléolos raramente presentes ou indefinidos e membrana nuclear bem visível com discreta irregularidade. Essas células apresentam-se isoladas ou agrupadas com boa definição celular. Pode ser observado efeito citopático compatível com infecção pelo papilomavírus humano (HPV), com presença de coilócitos, disqueratose, discariose e bi ou multinucleação.

\section{NIC 2}

Presença de células com relação nucleocitoplasmática aumentada, com formas variadas, carioteca espessada e irregular, cromatina irregular e hipercromática. As células afetadas são superficiais, intermediárias ou parabasais; apresentam-se isoladas, raramente em grupos; são arredondadas ou ovais, fusiformes ou bizarras.

\section{NIC 3}

Presença de células com contornos nucleares irregulares, de menor tamanho que os das células observadas em NIC 2 e com hipercromasia nuclear evidente. Encontramse isoladas ou em agrupamentos sinciciais, com tamanho aproximado de uma célula parabasal e formato redondo, oval, bizarro ou fusiforme. Seu núcleo ocupa dois terços da área total da célula; a carioteca é espessa.

\section{Carcinoma escamoso invasor}

Presença de células isoladas ou agrupadas, com distribuição irregular da cromatina, variação acentuada no tamanho e nos tipos celulares (células caudadas, em forma de girino e fusiformes), diátese tumoral.

\section{Adenocarcinoma}

Presença de células glandulares com aumento da relação nucleocitoplasmática, hipercromasia, nucléolos evidentes e diátese tumoral.

Todos os casos foram triados às cegas pelos citopatologistas e por profissionais paramédicos com experiência mínima de dez anos. O sistema SISCOLO (monitoramento externo) compara o resultado original com o da revisão, gerando um relatório dos casos discordantes.

Em relação aos critérios utilizados para avaliação, tomouse como base primeiramente os estabelecidos pelo Ministério da Saúde/Instituto Nacional do Câncer (MS/INCA), em que são considerados casos discordantes aqueles com implicação em mudança de conduta clínica, como:

Negativo $\leftrightarrow$ ASCUS/AGUS/HPV/NIC $1 \leftrightarrow$ NIC 2/

NIC 3/carcinoma

\section{Resultados}

No presente estudo não foram observados casos de falso negativo e falso positivo. A freqüência de concordância foi 86,62\%; a de discordância de um grau, $12,42 \%$; e a de discordância de mais de um grau, 0,96\%.

A freqüência dos diagnósticos dos laboratórios de origem e revisor está na Tabela 1. A Tabela 2 apresenta a avaliação da amostra referente à representatividade da JEC.

Freqüência dos diagnósticos dos

Tabela 1 laboratórios de origem e revisor

\begin{tabular}{lcc}
\hline Diagnóstico & $\begin{array}{c}\text { Origem } \\
n\end{array}$ & $\begin{array}{c}\text { Revisor } \\
n\end{array}$ \\
Negativo & 614 & 650 \\
ASCUS/AGUS & 93 & 39 \\
NIC 1 & 114 & 91 \\
NIC 2 & 8 & 13 \\
NIC 3 & 4 & 3 \\
Ca invasor & 1 & - \\
Insatisfatório & 3 & 41 \\
Total & 837 & 837 \\
\hline
\end{tabular}




\begin{tabular}{|c|c|c|}
\hline $\begin{array}{l}\text { Distribui } \\
\text { quanto à }\end{array}$ & \multicolumn{2}{|c|}{$\begin{array}{l}\text { Distribuição dos casos revisados } \\
\text { quanto à amostragem }\end{array}$} \\
\hline Amostra & $n$ & $\%$ \\
\hline Satisfatória & 196 & 23,4 \\
\hline Satisfatória mas limitada & 595 & 71,1 \\
\hline Insatisfatória & 42 & 5 \\
\hline Sem avaliação & 4 & 0,5 \\
\hline Total & 837 & 100 \\
\hline
\end{tabular}

\section{Discussão}

Como método laboratorial, o teste de Papanicolaou, apesar de críticas e limitações, efetivamente contribui para o controle de câncer de colo uterino. Existem contínuas e fundamentadas críticas quanto à sua sensibilidade, que gera uma quantidade significativa e indesejável de casos falsos negativos ${ }^{(14)}$. Além disso, há uma certa quantidade de casos com alterações citomorfológicas de resolução diagnóstica imprecisa, o que leva a polêmicas quanto à reprodutibilidade do método sob determinadas circunstâncias ${ }^{(5)}$.

$\mathrm{Na}$ avaliação de Neto et al. ${ }^{(9)}$, a citologia oncótica como método de rastreamento no Programa Nacional de Combate ao Câncer do Colo do Útero permitiu elevados índices de falsos negativos $(27,3 \%)$ e falsos positivos $(12,5 \%)$. Nessa condição, a colposcopia mostrou-se procedimento importante e indispensável nas condutas terapêuticas a serem adotadas.

Pelos resultados obtidos em nossos estudos, verificamos que os critérios diagnósticos dos laboratórios de origem e revisor foram similares, com bom índice de concordância
(86,62\%), não sendo observados casos de falsos negativos e falsos positivos.

A preocupação com os elevados índices de falsos negativos e falsos positivos na prevenção do câncer de colo uterino é mundial. É importante que medidas imediatas de controle de qualidade sejam adotadas para minimizar essa problemática $^{(3,4,7)}$.

Para isso, é necessário que os laboratórios de citopatologia disponham de:

- programa de controle de qualidade interno;

- programa de avaliação de controle de qualidade externo;

- permanente programa de treinamento/reciclagem;

- monitoramento de procedimentos de coleta, fixação e transporte de material citológico;

- laboratório revisor indicado por órgão competente ou laboratório de referência da região.

Diante de várias estratégias já estudadas, o rescreening rápido e a revisão de $100 \%$ dos casos são válidos para controle de qualidade interno ${ }^{(9)}$. A análise dos resultados de nosso estudo demonstra que o modelo utilizado é adequado para avaliação e monitoração do desempenho de laboratórios da rede de saúde pública, sendo possível sua implantação gradual em todo o território nacional(7). A utilização de seus resultados pode orientar medidas de educação continuada e padronização de critérios diagnósticos visando a implementação do controle de qualidade interno dos laboratórios. Quando pensamos em monitoramento externo, temos que levar em conta o volume de exames que irá ser avaliado(4). A estratégia adotada pelo Ministério da Saúde em todo território nacional parece ser hoje a mais apropriada.

\section{Referências}

I. ALVES, V. A. F. et al. Programa de controle de qualidade em citologia ginecológica do Instituto Adolfo Lutz: estratégias e análise crítica dos resultados de sua implantação piloto. AMB Rev Assoc Med Bras, v. 37, n. I, p. 36-42, 1991.

2.ANDERSON, G. H. et al.A comprehensive internal quality control system for a large cytology laboratory. Acta Cytol, v. 31 p. 895-9, 1987.

3. BRANCA, M. et al. Members of the working group for external quality control in cervical cytopathology. Reliability and accuracy in reporting CIN in 14 laboratories: developing new indices of diagnostic variability in an interlaboratory study. Acta Cytol, v. 42, p. 1370-6, 1998.
4. DAVEY, D. D.; MCGOOGAN, E.; SOMRAK, T. M. Competency assessment and proficiency testing. Acta Cytol, v. 44, p. 93943,2000

5. FAGUNDES, M. C. S. et al. Amostra inadequada em screening de esfregaços cervicovaginais: as principais causas. Laes \& Haes, v. 128, p. 94-100, 2000.

6. FARRELL, D. J. et al. Rapid screening of cervical smears as a method of internal quality control: for how long should we rescreen? Acta Cytol, v. 4I, n. 2, p. 25I-60, 1997.

7. FRABLE, W. J. et al. Medicolegal - IAC Task Force Summary. Acta Cytol, v. 42, n. I, p. 76-119, 1998. 
8. MODY, D. R.; DAVEY, D. D.; BRANCA, M. Quality assurance and risk reduction guidelines. Acta Cytol, v. 44, n. 4, p. 496-507.

9. NETO, A. R. et al. Avaliação dos métodos empregados no Programa Nacional de Combate ao Câncer do Colo Uterino do Ministério da Saúde. RBGO, v. 23, n. 4, p. 209|5, 200|.

10. RENSHAW,A.A. et al. Performance characteristics of rapid (30second) prescreening. Implications for calculating the falsenegative rate and comparison with other quality assurance technique. Am J Clin Pathol, v. I I I, p. 5 17-22, 1999.
I I. SILVA FILHO,A. M.; LONGATTO FILHO,A. Colo uterino e vagina: processos inflamatórios (aspectos histológicos, citológicos e colposcópicos). I ed. Rio de Janeiro: Editora Revinter, 2000. p. 35-54.

12.TABBARA, S. O.; SIDAW, M. K. Evaluation of the $10 \%$ rescreen of negative gynecologic smears as a quality assurance measure. Diagn Cytopathol, v. I4, p. 84-6, 1996.

I3.WALTON, R. J. Cervical cancer screening programsV. Conclusions and recommendation of the task force. Can Med Assoc J, v. I I4, n. I I, p. 1003-33, 1976. 